\title{
Review
}

Journal of Innate

Immunity
J Innate Immun 2015;7:563-571

DOI: $10.1159 / 000382019$
Received: January 17, 2015

Accepted after revision: April 1, 2015

Published online: April 30, 2015

\section{Human Cytokine-Induced Memory-Like Natural Killer Cells}

\author{
Melissa M. Berrien-Elliott Julia A. Wagner Todd A. Fehniger \\ Department of Medicine, Division of Oncology, Washington University School of Medicine, St. Louis, Mo., USA
}

\section{Key Words}

Natural killer cells · Innate memory · Immunotherapy ·

Cytokine $\cdot$ Cytokine receptor $\cdot \mathrm{IL}-12 \cdot \mathrm{IL}-15 \cdot \mathrm{IL}-18$

\begin{abstract}
Natural killer (NK) cells are innate lymphoid cells that are important for host defense against infection and mediate antitumor responses. Recent reports from several laboratories have identified that NK cells can remember a prior activation event and consequently respond more robustly when restimulated, a property termed innate NK cell memory. NK cell memory has now been identified following hapten exposure, viral infection, and combined cytokine preactivation with IL-12, IL-15, and IL-18. Many questions in the field remain regarding the cellular and molecular mechanisms regulating memory NK cells and their responses, as well as their formation and function in mice and humans. Here we review our current understanding of cytokine-induced memorylike (CIML) NK cells that are generated by combined preactivation with IL-12, IL-15, and IL-18. These cells exhibit enhanced NK cell effector functions weeks after the initial cytokine preactivation. Further, we highlight the preclinical rationale and ongoing therapeutic application of CIML NK cells for adoptive immunotherapy in patients with hematologic malignancies.

(c) 2015 S. Karger AG, Basel
\end{abstract}

(C) 2015 S. Karger AG, Basel

$1662-811 X / 15 / 0076-0563 \$ 39.50 / 0$

\section{Introduction}

The mammalian immune system is comprised of both innate and adaptive arms. The innate arm constitutes a host's first line of defense against pathogens, and consists of cells that express germ-line encoded receptors that recognize conserved pathogen-associated molecular patterns $[1,2]$. The innate immune response was not thought to be substantially altered by repeated exposure to the same challenge. In contrast, the adaptive immune system provides long-lasting immunity against pathogens via antigen-specific receptors and adaptive immune memory $[3,4]$. During their initial activation, $\mathrm{T}$ and $\mathrm{B}$ cells clonally expand in response to their cognate antigen. Nearly $90 \%$ of these responding cells undergo activation-induced apoptosis. The persisting pool of long-lived cells responds more robustly upon subsequent exposure to their cognate antigen, thus exhibiting a memory response [5]. For example, memory T cells exhibit increased proliferation, cytokine production, and cytotoxicity during recall responses, thereby more rapidly eliminating subsequent infections by the same pathogen. Thus, with repeated exposure to a specific pathogen, adaptive immune responses are boosted and can provide the host with lifelong antigen-specific immunity [6].

Natural killer (NK) cells are innate lymphoid cells critical for host defense against viruses and malignant cells [7-11]. This host protection relies on several important

\section{KARGER 125}

E-Mail karger@karger.com www.karger.com/jin 
NK cell functions. First, NK cells secrete cytokines and chemokines that activate, direct, and modify the host immune response. The prototypical cytokine released by NK cells is interferon- $\gamma$ (IFN- $\gamma$ ). IFN- $\gamma$ shapes T-cell immune responses, stimulates macrophages, upregulates MHC class I expression on antigen presenting cells, and decreases the proliferation of virally infected and malignant cells [7]. Second, NK cells are cytotoxic, with their ability to kill target cells mediated primarily by perforin and granzyme B, which are stored in cytotoxic granules [7]. These granules are exocytosed upon recognition of a target cell, a process that is tightly regulated to prevent indiscriminate killing [12].

Human NK cells originate in the bone marrow, differentiate in peripheral lymphoid tissue [13, 14], and are distinguished from other lymphoid cells by the absence of the T- and B-cell-specific markers (e.g. CD3 and CD19), and the presence of CD56 [7]. NK cells express a repertoire of germ-line encoded activating and inhibitory receptors that control their responses [15]. There are a variety of activating receptors (e.g. NKG2D and NKp46) that recognize ligands expressed on stressed, infected, or transformed cells, or antibody-opsonized targets (CD16/ Fc $\gamma$ RIIIa). Inhibitory signals arise from two main receptor families, killer cell immunoglobulin-like (KIR) and C-type lectin receptors (i.e. CD94-NKG2A), which recognize MHC class I or class I-like molecules [16]. There are two distinct human NK cell subsets identified phenotypicallybased on their surface density of CD 56: CD 56 $6^{\text {bright }}$ and CD $56^{\mathrm{dim}}$. CD $56^{\mathrm{dim}} \mathrm{NK}$ cells represent the majority of the NK cell population in the peripheral blood (80-95\%) and are specialized to respond to NK cell receptor-based stimulation that results in potent killing and cytokine secretion [7, 17-19]. In contrast, CD56 $6^{\text {bright }} \mathrm{NK}$ cells, which are the major subset of NK cells in secondary lymphoid tissues, respond primarily to cytokine receptor-based stimulation to produce abundant cytokines and chemokines, but are poorly cytotoxic at rest. The distinct biology of CD56 $6^{\text {bright }}$ and CD56 $6^{\text {dim }} \mathrm{NK}$ cells is also reflected in differing complements of cell surface cytokine, chemokine, homing, integrin, and NK receptors $[19,20]$. For example, CD56 ${ }^{\mathrm{dim}} \mathrm{NK}$ cells express both KIR and CD94NKG2A inhibitory receptors, whereas CD56 bright NK cells primarily express CD94-NKG2A. Additionally, CD16 (Fc $\gamma$ RIIIa) is expressed on most CD56 ${ }^{\text {dim }} \mathrm{NK}$ cells and a subset of CD56 $6^{\text {bright }} \mathrm{NK}$ cells, allowing for antibodydependent cellular cytotoxicity [19]. Within CD $56^{\mathrm{dim}} \mathrm{NK}$ cells, distinct functional subsets may be defined by maturation stage with less mature cells expressing CD94 and CD62L, but lacking CD57 [21-23]. The current view of human NK cell maturation is that CD56 bright (stage IV) NK cells differentiate into CD56 ${ }^{\mathrm{dim}}$ (stage V) NK cells [24]. However, the developmental relationship between the two remains controversial and it is possible that these subsets have distinct precursors [25]. Regardless, CD$56^{\text {bright }}$ and $\mathrm{CD} 56^{\mathrm{dim}}$ represent distinct human NK cell subsets with differing physiological roles.

Traditionally, NK cells have been classified as innate effectors since they do not rearrange their DNA to express a specific, dominant activating receptor. More recently, however, evidence has emerged that challenges this paradigm, demonstrating that NK cells display properties of immunologic memory. This review summarizes mouse and human studies that have defined the concept of innate $\mathrm{NK}$ cell memory, with particular focus on human cytokine-induced memory-like (CIML) NK cells. Furthermore, we describe ongoing translational studies utilizing CIML NK cells for the treatment of hematologic malignancies.

\section{NK Cell Memory}

Several reports have described that innate NK cell-mediated memory can be generated in response to certain haptens and viruses, as well as following combined cytokine activation (fig. 1) [26]. NK cell memory was first described in response to two haptens, 2,4-dinitrofluorobenzene and oxazolone [27]. Mice lacking T and B cells, but not NK cells, exhibited specific contact hypersensitivity responses to these haptens that persisted for at least 4 weeks. Furthermore, adoptive transfer of NK cells from sensitized donors resulted in contact hypersensitivity responses in the recipients upon reexposure to that same hapten [27]. These NK cell memory responses to haptens are dependent on expression of CXCR6, a chemokine receptor critical for intrahepatic survival and homeostasis [28]. The molecular mechanism that explains the varying, yet specific hapten recall responses remains poorly understood.

A subsequent study demonstrated that murine NK cells exhibit a memory-like adaptive response following murine cytomegalovirus (MCMV) infection [29]. Specifically, NK cells bearing the activating receptor Ly $49 \mathrm{H}$, which recognizes the MCMV-expressed ligand m157, proliferate in response to MCMV infection. After a contraction phase, $\mathrm{Ly} 49 \mathrm{H}^{+} \mathrm{NK}$ cells persist for months. Upon isolation and ex vivo restimulation with plate-bound agonistic antibodies against NK1.1 or Ly49H, these cells rapidly degranulated and produced cytokines more robustly than MCMV-inexperienced NK cells [29]. Additionally, 


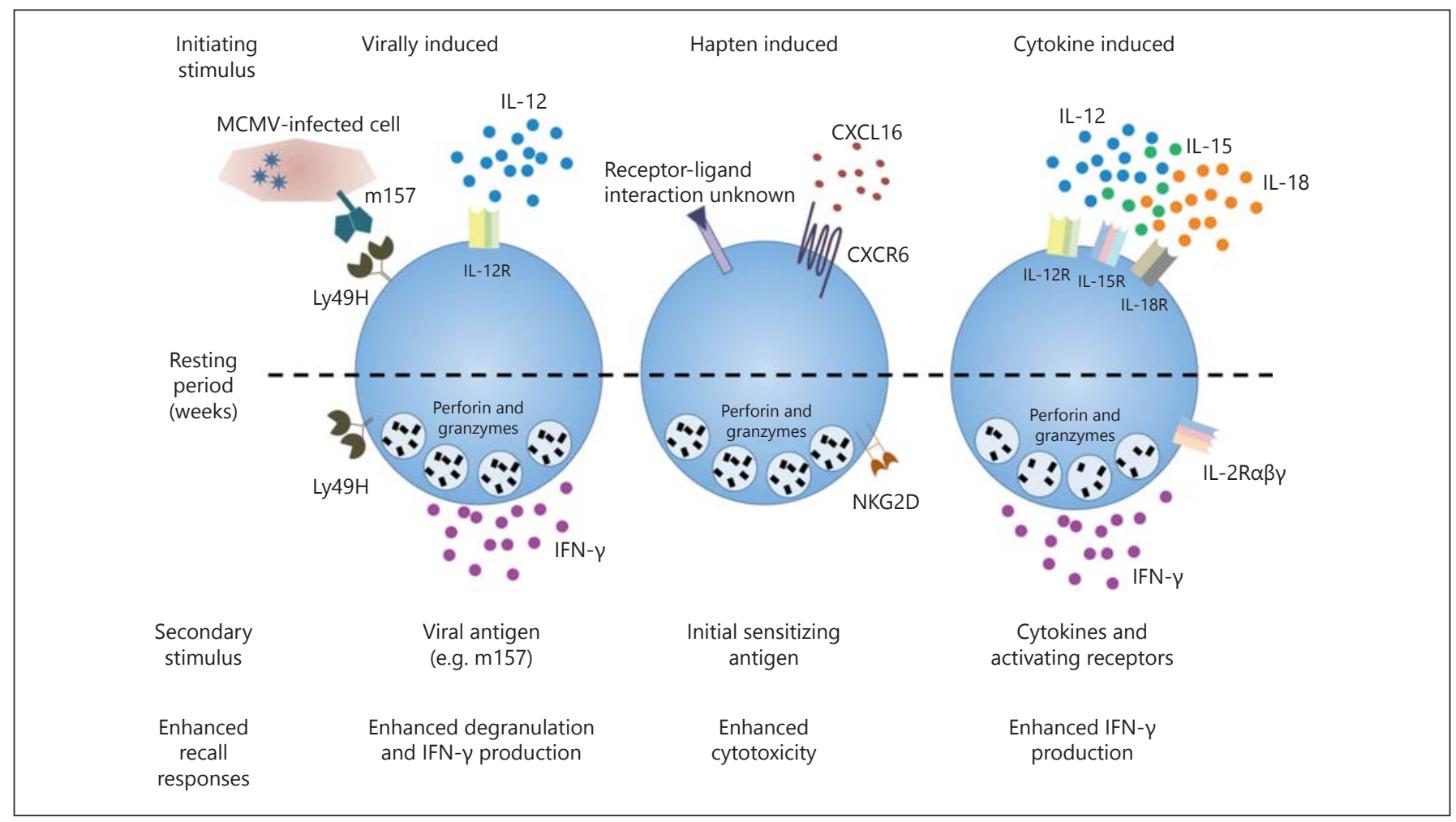

Fig. 1. Overview of murine memory NK cells. Three types of stimuli induce NK cell memory in mice: viral infection, hapten sensitization, and cytokines. Weeks after initial activation, memory-like NK cells exhibit enhanced recall responses during subsequent reactivation. The resulting NK cells are long-lived and display enhanced responsiveness, which is maintained despite multiple rounds of division. adoptive transfer of these memory NK cells, compared to naïve, provided protective immunity to newborn mice, which lack mature NK cells and are thus highly susceptible to MCMV infection [29]. Subsequent studies demonstrated that virus-induced NK cell memory is dependent on the proinflammatory cytokine IL-12 [30].

Human studies have demonstrated that infection with cytomegalovirus (CMV) [31-33], hantavirus [34], or chikungunya virus [35] results in an imprinted NK cell receptor repertoire with an increased frequency of $\mathrm{NKG} 2 \mathrm{C}^{+} \mathrm{NK}$ cells. In the case of CMV, NKG2 $\mathrm{C}^{+} \mathrm{NK}$ cells expanded during acute infection and persisted for up to a year [36]. Additionally, these $\mathrm{NKG}_{2} \mathrm{C}^{+} \mathrm{NK}$ cells produced significantly more IFN- $\gamma$ in response to target cells than NKG2C $\mathrm{C}^{-} \mathrm{NK}$ cells [31]. Furthermore, adoptively transferred NKG2C ${ }^{+} \mathrm{NK}$ cells from CMV-seropositive persons displayed enhanced effector function against a secondary CMV challenge in the recipient compared to NKG2C ${ }^{+} \mathrm{NK}$ cells from CMV-seronegative individuals [36]. Recent work has demonstrated that the combination of CMV-infected fibroblasts plus IL-12-producing monocytes results in the expansion of $\mathrm{NKG}_{2} \mathrm{C}^{+} \mathrm{NK}$ cells in vitro [37]. In addition, the induction of CD25 and the high-affinity IL-2R $\alpha \beta \gamma$ contribute to the expansion in this in vitro model, which also depends on NKG2C and HLA$\mathrm{E}$ interactions. The precise parallels between MCMVinduced memory NK cells and $\mathrm{NKG}_{2} \mathrm{C}^{+}$human NK cells in people with latent CMV are incompletely understood.

\section{Mouse CIML NK Cells}

At the same time virus-induced NK cell memory was reported, Cooper et al. [38] discovered that murine NK cells preactivated with cytokines exhibit memory-like properties. In this report, NK cells were preactivated overnight with IL-12, IL-15, and IL-18, or IL-15 alone as a control, and then adoptively transferred into syngeneic Rag$1^{-/-}$recipients. Although a majority of the preactivated NK cells produced IFN- $\gamma$ at the time of adoptive transfer, after weeks of rest in the recipients, IFN- $\gamma$ production returned to baseline, undetectable levels. Furthermore, preactivated NK cells proliferated extensively in vivo, whereas control NK cells did not $[38,39]$. After resting in vivo, 
the CIML (IL-12-, IL-15-, and IL-18-preactivated) NK cells were phenotypically similar to control NK cells as they expressed similar levels of CD69, CD11b, CD27, and B220, as well as the cytokine receptors CD122, IL12R $\beta 1$, IL-15Ra, and CD127 [38]. Likewise, murine CIML NK cell cytotoxicity was similar to that of control NK cells, as they expressed comparable levels of granzyme B and lysed target cells comparably to controls in vitro [38, 39]. However, CIML NK cells displayed enhanced IFN- $\gamma$ production upon activating receptor ligation or cytokine restimulation compared to controls [38]. This enhanced ability to produce IFN- $\gamma$ was cell-intrinsic and persisted following cell division, including homeostatic proliferation in NK cell-deficient recipients $[38,39]$.

The underlying mechanisms that result in more abundant IFN- $\gamma$ expression by CIML NK cells upon restimulation has not yet been elucidated. Notably, it has been demonstrated that both control and CIML NK cells express similar levels of IFN- $\gamma$ transcript mRNA, indicating that the enhanced functionality of CIML NK cells is not due to a simple alteration in IFN- $\gamma$ transcription or mRNA stability [38, 39]. Further studies into these mechanisms are warranted.

Following this initial report, Ni et al. [40] demonstrated that adoptively transferred murine IL-12-, IL-15-, and IL-18-preactivated NK cells display enhanced effector function against established tumors in vivo. Syngeneic NK cells were preactivated with IL-12, IL-15, and IL-18 or IL-15 alone for $16 \mathrm{~h}$ and adoptively transferred into recipient mice bearing MHC class I-deficient RMA-S lymphoma or B16-Rae1ع melanoma cell lines. Following transfer, the preactivated NK cells proliferated rapidly in an IL-2-dependent manner. Furthermore, significantly reduced tumor growth and prolonged survival was observed in recipient mice treated with IL-12-, IL-15-, and IL-18-preactivated NK cells compared to mice treated with control NK cells [40]. Irradiation of the mice was also necessary for the IL-12-, IL-15-, and IL-18-preactivated NK cells' persistence and antitumor efficacy [40]. Thus, mouse NK cells preactivated with IL-12, IL-15, and IL-18 exhibited enhanced functionality months following adoptive transfer and were able to mediate more effective in vivo antitumor responses.

\section{Human CIML NK Cells}

Human NK cells also exhibit CIML activity (fig. 2) [41]. Purified human NK cells from normal donors were stimulated with various combinations of IL-12, IL-15, and
IL-18 for $16 \mathrm{~h}$. Following removal of these cytokines, the cells were rested in vitro with a low dose of IL-15 as a survival factor. After 1-3 weeks of rest, NK cells preactivated with IL-12, IL-15, and IL-18 or double combinations of IL-12, IL-18, and IL-15 exhibited increased IFN- $\gamma$ production after restimulation with cytokines or tumor target cells compared to control cells incubated with IL-12, IL-15, or IL-18 alone [41]. Because IL-2 and IL-15 both signal through the IL15R $\beta \gamma c$, it is likely that preactivation with intermediate levels IL-2 combined with IL-12 and IL-18 would result in memory-like NK cells; however, this has not been experimentally determined. Although gating on $\mathrm{CD} 56^{\text {bright }}$ versus $\mathrm{CD} 56^{\mathrm{dim}}$ cell populations, particularly after $>2$ weeks in vitro, can be challenging, we observed enhanced IFN- $\gamma$ production following cytokine preactivation by both $\mathrm{CD} 56^{\text {bright }}$ and $\mathrm{CD} 56^{\mathrm{dim}} \mathrm{NK}$ cell subsets, with a particularly robust effect on the CD56 ${ }^{\text {bright }}$ CIML NK cell subset [41]. Human CIML NK cells proliferated extensively, but maintained their capacity for enhanced recall responses even after multiple rounds of cell division tracked by CFSE dilution. Similar to mice, IFN- $\gamma$ mRNA transcript levels did not differ between CIML and control NK cells, suggesting that a simple difference in the IFN- $\gamma$ mRNA pool was not the mechanism responsible for enhanced IFN- $\gamma$ [41]. However, in contrast to murine CIML NK cells, phenotypic differences were identified between preactivated NK cells and controls. Specifically, CIML NK cells had increased CD94, NKG2A, NKp46, and CD69 surface expression. Further, CIML NK cells were associated with reduced KIR and CD57 expression [41]. As a population, enhanced IFN- $\gamma$ production by CIML NK cells was associated with expression of CD94, NKG2A, NKG2C, and CD69, and with the lack of KIR and CD57. These findings may assist in identifying CIML NK cells populations in vivo.

Additionally, human CIML NK cells were also shown to be responsive to low concentrations of IL-2, which is critical for promoting their function and proliferation in vivo [42]. The IL-2 receptor is composed of either intermediate-affinity (IL-2R $\beta \gamma$ ) or high-affinity (IL-2R $\alpha \beta \gamma$ ) forms that are ligated by nanomolar and picomolar concentrations of IL-2, respectively [43, 44]. CD25 (IL-2Ra) is a critical component required to form the high-affinity receptor. CD25 is constitutively expressed at low levels by $\mathrm{CD} 56^{\text {bright }}$, but not $\mathrm{CD} 56^{\mathrm{dim}} \mathrm{NK}$ cells, making CD56 ${ }^{\text {bright }}$ NK cells more responsive to low-dose IL-2 [7, 45]. Recently, it has been demonstrated that CD56 $6^{\text {bright }}$ and CD56 ${ }^{\mathrm{dim}}$ CIML NK cells markedly upregulated CD25 in response to combined cytokine preactivation, resulting in the formation of a functional high-affinity 


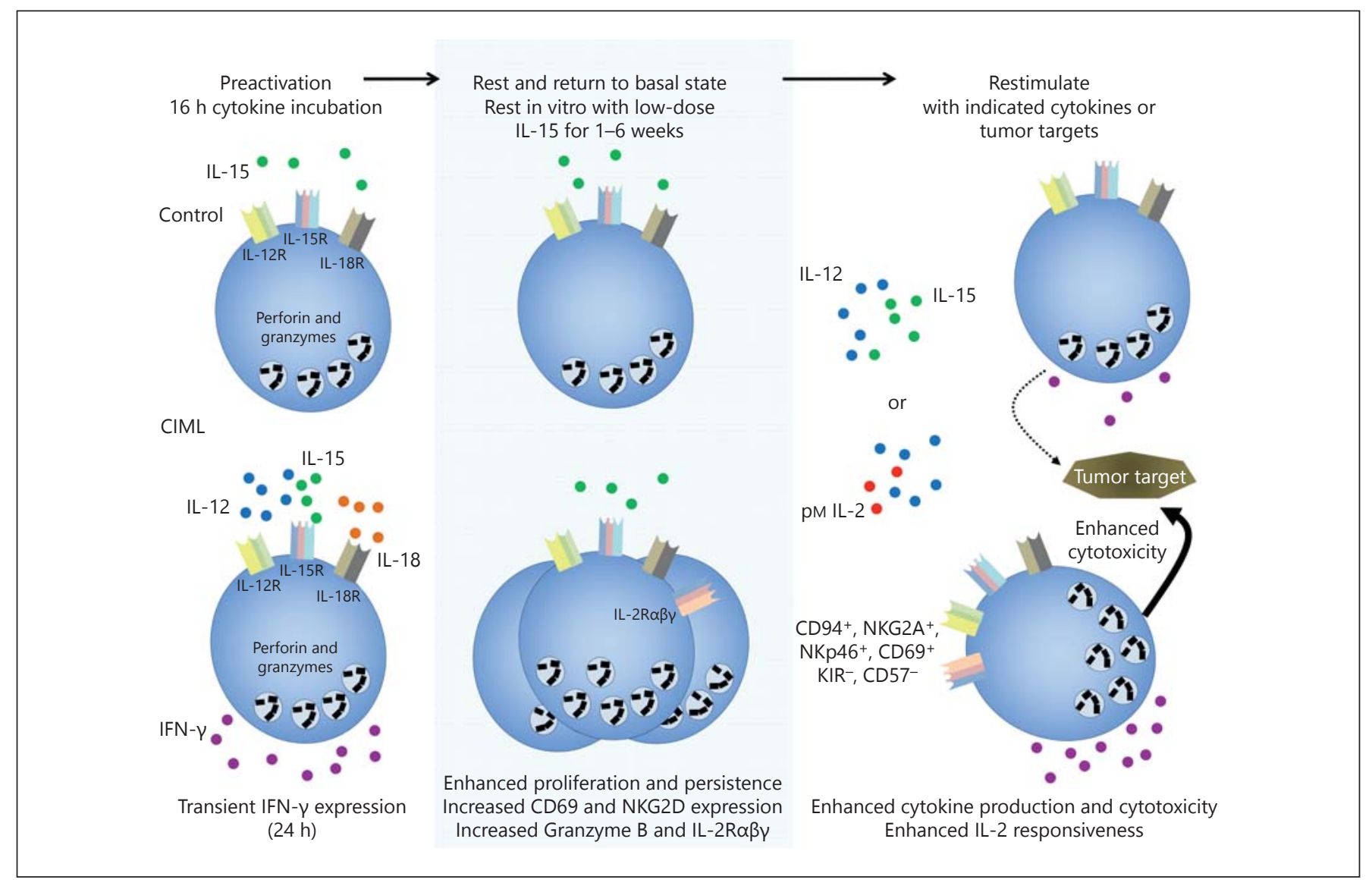

Fig. 2. Schema of human CIML NK cell generation. Purified NK cells are preactivated with IL-15 (control) or IL-12, IL-15, and IL18 (CIML) for $16 \mathrm{~h}$, washed, and rested for $>7$ days with low-dose IL-15 to support survival. After the initial activation, CIML NK

IL-2 receptor (IL-2R $\alpha \beta \gamma$ ) that is ligated by picomolar concentrations of IL-2 [42]. In this study, provision of picomolar concentrations of IL-2 resulted in the proliferation of IL-12-, IL-15-, and IL-18-preactivated NK cells both in vitro and in vivo. Further, CIML NK cell IFN $-\gamma$ production and cytotoxic capacity were enhanced by provision of picomolar concentrations of IL-2. These data suggest that combined cytokine preactivation results in CIML NK cells that are well adapted to utilize T cell-derived IL-2, or low-dose exogenous rhIL-2, to support their survival and functionality.

Recently, preclinical studies have shown that human CIML NK cells also have substantial potential as antileukemia cellular therapy [46]. Human CIML NK cells have exhibited enhanced IFN- $\gamma$ production when restimulated (triggered) with leukemia cell lines or acute myeloid leukemia (AML) blasts in vitro. Additionally, granzyme B protein abundance and cytotoxicity were increased in hu- cells express IFN $-\gamma$, which returns to baseline levels by $24 \mathrm{~h}$. CIML NK cells display increased IFN- $\gamma$ production and enhanced cytotoxicity after restimulation, compared to control NK cells. pM = Picomolar.

man CIML, compared to control NK cells from the same donors [46]. However, enhanced degranulation in response to tumor targets was not observed in human CIML NK cells compared to controls [41], paralleling what has been observed in mice [38]. Thus, the enhanced antileukemic function of human CIML NK cells likely relies on a combination of increased cytotoxicity as well as enhanced IFN- $\gamma$ production, but the precise effector mechanism important for antileukemia responses have not been experimentally defined. CIML NK cells may also be engrafted into immunodeficient NOD-SCID $-\gamma \mathrm{c}^{-/-}$mice (NSG) mice and supported by low-dose rhIL-2. In this NSG xenograft model, CIML NK cells exhibited superior in vivo persistence, compared to control NK cells, following adoptive transfer [42]. Ex vivo analysis of CIML NK cells after transfer and rest in NSG mice revealed the memory-like phenotype with increased IFN- $\gamma$ production following restimulation. Finally, CIML NK cells 


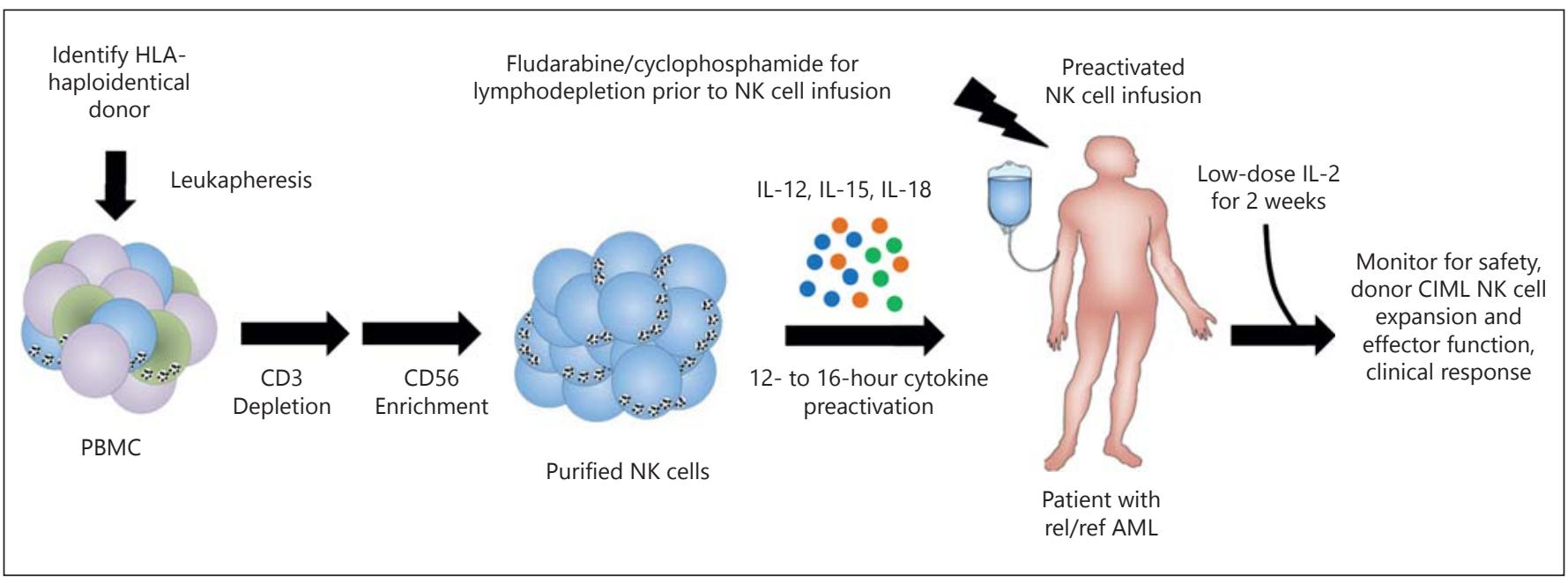

Fig. 3. CIML NK cell adoptive immunotherapy for cancer. CIML NK cell adoptive immunotherapy clinical strategy schema.

exhibited a superior ability to control AML cells when transferred into leukemia-bearing NSG mice. These results, taken together with murine CIML NK cell cancer immunotherapy studies, provide the scientific rationale that cytokine preactivation with IL-12, IL-15, and IL-18 and administration of low-dose IL-2 will enhance the antileukemic effector function of adoptively transferred NK cells in humans.

\section{CIML NK Cells: From Bench to Bedside}

NK cells can recognize and respond to malignant tumor cells and are an important immunotherapeutic effector for cancer, particularly hematologic cancers [47-51]. The biology of NK cells makes them an outstanding candidate for anticancer therapy. First, unlike allogeneic $\mathrm{T}$ cells, NK cells do not induce graft-versus-host disease, and in fact may mediate graft-versus-host-disease protection $[52,53]$. Second, some cancer cells can be recognized by allogeneic NK cells, and based on progress in NK cell receptor biology, haploidentical donors may be chosen to maximize the NK cell versus leukemia response. In particular, Ruggeri et al. [53] first showed that KIR-ligand mismatch, resulting in donor NK cell alloreactivity against recipient leukemia, markedly reduced AML relapse in patients receiving HLA-haploidentical hematopoietic stem cell transplants. Consistent with these findings, donor KIR haplotype is predictive of AML relapse risk in the setting of allogeneic transplantation $[54,55]$. The antileukemic effector function of enriched NK cells using adoptive transfer has also been demonstrated in humans [56, 57]. For example, Miller et al. [56] transferred enriched NK cells (CD3- and CD19-depleted peripheral blood mononuclear cells) from haploidentical donors into patients with poor prognosis AML. The provision of enriched NK cells in this fashion resulted in complete remissions in about $30 \%$ of patients, but of relatively short duration. Rubnitz et al. [57] administered allogeneic NK cells to pediatric AML patients already in complete remission, and demonstrated safety and NK cell detection after the infusion. These and other studies have demonstrated that allogeneic NK cells may be safely administered to leukemia patients, but a limited complete remission rate and duration of response remain a barrier in the field. Therefore, approaches that enhance the functionality, expansion, and survival of adoptively transferred NK cells are needed.

Based on the basic and preclinical reports described above, we hypothesized that combined cytokine preactivation with IL-12, IL-15, and IL-18 resulting in CIML NK cell generation would be a safe and potentially effective strategy to translate NK cell memory into improved antiAML NK cell responses. This hypothesis is currently being explored in a clinical trial of adoptively transferred allogeneic CIML NK cells in patients with relapsed or refractory AML (fig. 3, NCT01898793). For this first-in-human NK cell study, rel/ref AML patients are lymphodepleted using fludarabine and cyclophosphamide [56], followed by a single CIML NK cell infusion. After CIML NK cell transfer, low-dose rhIL-2 is administered for 14 days to support CIMK NK cell survival, expansion, and function based on the expression of the induced CD25 and 
thus the high-affinity IL-2R $\alpha \beta \gamma$ [58]. The clinical grade CIML NK cell product is generated from a single haploidentical donor leukapheresis, followed by CD3 depletion and CD56 enrichment using magnetic selection. This approach typically results in $\geq 90 \% \mathrm{CD}^{2} 6^{+} \mathrm{CD}^{-} \mathrm{NK}$ cells, which are activated for $12-16 \mathrm{~h}$ in rhIL-12, rhIL-15, and rhIL-18 in a GMP-compliant biological therapy facility. Prior to infusion, all cytokines are washed from the NK cell product to prevent transfer of proinflammatory agents to the patient. Correlative laboratory analyses are tracking donor CIML NK cell persistence, expansion, proliferation, and memory-like functionality at various time points following infusion. If this CIML NK cell adoptive therapy appears safe, clinical testing will be expanded to a larger number of AML patients to evaluate efficacy. Since CIML NK cell express CD16/Fc $\gamma$ RIIIa, provision of therapeutic antibodies or newer bispecific NK cell reagents could potentially be included to enhance specificity and triggering against other types of cancer.

\section{Conclusions and Open Questions}

Cellular-based therapies are gaining traction in the cancer immunotherapy field, including the use of NK cells for the treatment of hematologic malignancies. Human CIML NK cells have a number of properties that may be beneficial for antitumor responses, including enhanced function against leukemia, expansion in vivo, and responsiveness to low-dose IL-2 [41, 42, 46]. A clinical trial is now underway to test the safety of IL-12, IL-15, and IL-18 cytokine preactivated NK cells, which may be a safe and effective means of improving NK cell anticancer functionality in the context of adoptive therapy. It is remarkable that discovery of CIML NK cells has moved from murine models to a first-in-human trial in 5 years. However, despite these translational advances, we have a limited understanding of the molecular mechanisms governing CIML NK cell differentiation and functionality. These are important questions in the memory NK cell field since understanding memory at the molecular and cellular level may lead to new strategies to further enhance antitumor functionality.

There are also many other questions that remain to be answered. How are CIML NK cells generated during a physiologic immune response to infection or cancer? How do enhanced CIML NK cell effector functions contribute to antitumor immunity? Do accessory cells such as dendritic cells or macrophages that produce proinflammatory cytokines induce CIML NK cell function? Do
CIML NK cells follow the rules of NK cell licensing, which prevents autoreactivity under homeostatic conditions [59]? Can CIML NK cells out-compete regulatory T cells for limiting amounts of IL-2, impeding a known brake on NK cell responses? What is the relationship of CIML NK cells to the $\mathrm{NKG}_{2} \mathrm{C}^{+}$imprinted memory-like NK cells that differentiate following CMV infection [31-33]?

The finding that virus-induced NK cell memory requires proinflammatory cytokines such as IL-12 [30, 37] suggests that cytokine receptor signaling may be a common pathway central to the generation of innate NK cell memory. Furthermore, the preservation of enhanced CIML NK cell functionality following multiple rounds of cell division $[38,41]$ suggests that epigenetic alterations may contribute to CIML NK cell differentiation and function. Indeed, epigenetic changes have been observed in cytokine-preactivated and $\mathrm{NKG} 2 \mathrm{C}^{+}$-imprinted human NK cells, reinforcing a potential role for epigenetic imprinting in NK cell memory [60-62]. An improved understanding of human CIML NK cell biology will be important to expand the clinical potential and spectrum of NK cell memory. We are hopeful that as our understanding of CIML NK cell biology expands, we will translate these findings to better outcomes for cancer patients.

\section{Acknowledgments}

We thank Drs. Megan Cooper, Rizwan Romee, Wayne Yokoyama, Anthony French, and all members of the Fehniger lab for insightful discussion. This work was supported by the Siteman Cancer Center/Cancer Frontier Fund at Washington University (T.A.F.), Gabrielle's Angel Foundation for Cancer Research (T.A.F.), R01AI102924 (T.A.F.) and the Washington University Institute of Clinical and Translational Sciences grants UL1 TR000448 and TL1 TR000449 from the National Center for Advancing Translational Sciences (J.A.W.).

References

1 Janeway CA Jr, Medzhitov R: Innate immune recognition. Annu Rev Immunol 2002;20: 197-216.

2 Mogensen TH: Pathogen recognition and inflammatory signaling in innate immune defenses. Clin Microbiol Rev 2009;22:240-273.

3 Cooper MD, Alder MN: The evolution of adaptive immune systems. Cell 2006;124: 815-822.

4 Vitetta ES, Berton MT, Burger C, Kepron M, Lee WT, Yin XM: Memory B and T cells. Annu Rev Immunol 1991;9:193-217.

5 Farber DL, Yudanin NA, Restifo NP: Human memory T cells: generation, compartmentalization and homeostasis. Nat Rev Immunol 2014;14:24-35. 
6 Kim C, Williams MA: Nature and nurture: Tcell receptor-dependent and $\mathrm{T}$-cell receptorindependent differentiation cues in the selection of the memory T-cell pool. Immunology 2010;131:310-317.

7 Caligiuri MA: Human natural killer cells. Blood 2008;112:461-469.

8 Vivier E, Tomasello E, Baratin M, Walzer T, Ugolini S: Functions of natural killer cells. Nat Immunol 2008;9:503-510.

9 Di Santo JP: Natural killer cells: diversity in search of a niche. Nat Immunol 2008;9:473475.

10 Vidal SM, Khakoo SI, Biron CA: Natural killer cell responses during viral infections: flexibility and conditioning of innate immunity by experience. Curr Opin Virol 2011;1:497512.

11 Orr MT, Lanier LL: Natural killer cell education and tolerance. Cell 2010;142:847-856.

12 Orange JS: Formation and function of the lytic NK-cell immunological synapse. Nat Rev Immunol 2008;8:713-725.

13 Freud AG, Caligiuri MA: Human natural killer cell development. Immunol Rev 2006;214: 56-72.

$14 \mathrm{Yu}$ J, Freud AG, Caligiuri MA: Location and cellular stages of natural killer cell development. Trends Immunol 2013;34:573-582.

15 Long EO, Sik Kim H, Liu D, Peterson ME, Rajagopalan S: Controlling natural killer cell responses: integration of signals for activation and inhibition. Annu Rev Immunol 2013;31: 227-258.

16 Lanier LL: NK cell receptors. Annu Rev Immunol 1998;16:359-393.

17 Maria A De, Bozzano F, Cantoni C, Moretta L: Revisiting human natural killer cell subset function revealed cytolytic $\mathrm{CD} 56^{\mathrm{dim}} \mathrm{CD} 16+$ NK cells as rapid producers of abundant IFN- $\gamma$ on activation. Proc Natl Acad Sci U S A 2011;108:728-732.

18 Fauriat C, Long EO, Ljunggren $\mathrm{H}$, Bryceson YT: Regulation of human NK-cell cytokine and chemokine production by target cell recognition. Blood 2010;115:2167-2176.

19 Cooper MA, Fehniger TA, Caligiuri MA: The biology of human natural killer-cell subsets. Trends Immunol 2001;22:633-640.

20 Romee R, Leong JW, Fehniger TA: Utilizing cytokines to function-enable human NK cells for the immunotherapy of cancer. Scientifica (Cairo) 2014;2014:205796.

21 Lopez-Vergès S, Milush JM, Pandey S, York VA, Arakawa-Hoyt J, Pircher H, et al: CD57 defines a functionally distinct population of mature NKcells in the human CD56 ${ }^{\mathrm{dim}} \mathrm{CD} 16+$ NK-cell subset. Blood 2010;116:3865-3874.

22 Yu J, Mao HC, Wei M, Hughes T, Zhang J, Park I, et al: CD94 surface density identifies a functional intermediary between the CD56 ${ }^{\text {bright }}$ and CD56 ${ }^{\text {dim }}$ human NK-cell subsets. Blood 2010;115:274-281.

23 Björkström NK, Riese P, Heuts F, Andersson $S$, Fauriat C, Ivarsson MA, et al: Expression patterns of NKG2A, KIR, and CD57 define a process of CD56 ${ }^{\mathrm{dim}}$ NK-cell differentiation uncoupled from NK-cell education. Blood 2010;116:3853-3864.

24 Chan A, Hong D, Atzberger A, Filer AD, Buckley CD, Mcmichael A, et al: CD56 ${ }^{\text {bright }}$ human NK cells differentiate into CD56 $6^{\mathrm{dim}}$ cells: role of contact with peripheral fibroblasts. J Immumol 2013;179:89-94.

25 Mace EM, Hsu AP, Monaco-Shawver L, Makedonas G, Rosen JB, Dropulic L, et al: Mutations in GATA2 cause human NK cell deficiency with specific loss of the CD56 ${ }^{\text {bright }}$ subset. Blood 2013;121:2669-2677.

26 Min-Oo G, Kamimura Y, Hendricks DW, Nabekura T, Lanier LL: Natural killer cells: walking three paths down memory lane. Trends Immunol 2013;34:251-258.

27 O'Leary JG, Goodarzi M, Drayton DL, Yu H, von Andrian UH: T cell- and B cell-independent adaptive immunity mediated by natural killer cells. Nat Immunol 2006;7:507-516.

28 Paust S, Gill HS, Wang B-Z, Flynn MP, Moseman EA, Senman B, et al: Critical role for the chemokine receptor CXCR6 in NK cell-mediated antigen-specific memory of haptens and viruses. Nat Immunol 2010;11:11271135 .

29 Sun JC, Beilke JN, Lanier LL: Adaptive immune features of natural killer cells. Nature 2009;457:557-561.

30 Sun JC, Madera S, Bezman NA, Beilke JN, Kaplan MH, Lanier LL: Proinflammatory cytokine signaling required for the generation of natural killer cell memory. J Exp Med 2012; 209:947-954

31 Foley B, Cooley S, Verneris MR, Pitt M, Curtsinger J, Luo X, et al: Cytomegalovirus reactivation after allogeneic transplantation promotes a lasting increase in educated NKG2C+ natural killer cells with potent function. Blood 2012;119:2665-2674.

32 Gumá M, Angulo A, Vilches C, Gómez-lozano N, Malats N, Lopez-Botet M: Imprint of human cytomegalovirus infection on the NK cell receptor repertoire. Blood 2004;104: 3664-3671.

33 Gumá M, Budt M, Sáez A, Brckalo T, Hengel $\mathrm{H}$, Angulo A, et al: Expansion of CD94/ $\mathrm{NKG} 2 \mathrm{C}+\mathrm{NK}$ cells in response to human cytomegalovirus-infected fibroblasts. Blood 2006; 107:3624-3631.

34 Björkström NK, Lindgren T, Stoltz M, Fauriat C, Braun M, Evander M, et al: Rapid expansion and long-term persistence of elevated NK cell numbers in humans infected with hantavirus. J Exp Med 2011;208:13-21.

35 Petitdemange C, Becquart P, Wauquier N, Béziat $\mathrm{V}$, Debré $\mathrm{P}$, Leroy EM, et al: Unconventional repertoire profile is imprinted during acute chikungunya infection for natural killer cells polarization toward cytotoxicity. PLoS Pathog 2011;7:e1002268.

36 Foley B, Cooley S, Verneris MR, Curtsinger J, Luo X, Waller EK, et al: Human cytomegalovirus (CMV)-induced memory-like NKG2C(+) $\mathrm{NK}$ cells are transplantable and expand in vivo in response to recipient CMV antigen. J Immunol 2012;189:5082-5088.
37 Rölle A, Pollmann J, Ewen E, Le VT, et al: IL12-producing monocytes and HLA-E control HCMV-driven NKG2C+ NK cell expansion. J Clin Invest 2014;124:5305-5316.

38 Cooper MA, Elliott JM, Keyel PA, Yang L, Carrero JA, Yokoyama WM: Cytokine-induced memory-like natural killer cells. Proc Natl Acad Sci U S A 2009;106:1915-1919.

39 Keppel M, Yang L, Cooper M: Murine NK cell intrinsic cytokine-induced memory-like responses are maintained following homeostatic proliferation. J Immumol 2013;190:47544762.

40 Ni J, Miller M, Stojanovic A, Garbi N, Cerwenka A: Sustained effector function of IL12/15/18-preactivated NK cells against established tumors. J Exp Med 2012;209:23512365.

41 Romee R, Schneider SE, Leong JW, Chase JM, Keppel CR, Sullivan RP, et al: Cytokine activation induces human memory-like NK cells. Blood 2012;120:4751-4760.

42 Leong JW, Chase JM, Romee R, Schneider SE, Sullivan RP, Cooper MA, et al: Preactivation with IL-12, IL-15, and IL-18 Induces CD25 and a functional high-affinity IL-2 receptor on human cytokine-induced memory-like natural killer cells. Biol Blood Marrow Transpl 2014;20:463-473.

43 Waldmann TA: The biology of interleukin-2 and interleukin-15: implications for cancer therapy and vaccine design. Nat Rev Immunol 2006;6:595-601.

44 Malek TR: The biology of interleukin-2. Ann Rev Immunol 2008;26:453-479.

45 Fehniger TA, Cooper MA, Nuovo GJ, Cella M, Facchetti F, Colonna M, et al: CD56 ${ }^{\text {bright }}$ natural killer cells are present in human lymph nodes and are activated by T cell-derived IL-2: a potential new link between adaptive and innate immunity. Blood 2003;101: 3052-3057.

46 Rosario M, Romee R, Schneider S, Wagner J, Berrien-Elliott M, Leong J, et al: Human cytokine-induced memory-like NK cells are active against myeloid leukemia in vitro and in vivo. Blood 2014;124:A1117.

47 Farag SS, Fehniger TA, Ruggeri L, Velardi A, Caligiuri MA: Natural killer cell receptors: new biology and insights into the graft-versus-leukemia effect. Blood 2002;100:19351947.

48 Ljunggren HG, Malmberg KJ: Prospects for the use of NK cells in immunotherapy of human cancer. Nat Rev Immunol 2007;7:329-339.

$49 \mathrm{Wu}$ J, Lanier LL: Natural killer cells and cancer. Adv Cancer Res 2003;90:127-156.

50 Velardi A, Ruggeri L, Mancusi A, Aversa F, Christiansen FT: Natural killer cell allorecognition of missing self in allogeneic hematopoietic transplantation: a tool for immunotherapy of leukemia. Curr Opin Immunol 2009;21:525-530.

51 Hsu KC, Dupont B: Natural killer cell receptors: regulating innate immune responses to hematologic malignancy. Semin Hematol 2005;42:91-103. 
52 Olson JA, Leveson-Gower DB, Gill S, Baker J, Beilhack A, Negrin RS: NK cells mediate reduction of GVHD by inhibiting activated, alloreactive T cells while retaining GVT effects. Blood 2010;115:4293-4301.

53 Ruggeri L, Capanni M, Urbani E, Perruccio K, Shlomchik WD, Tosti A, et al: Effectiveness of donor natural killer cell alloreactivity in mismatched hematopoietic transplants. Science 2002;295:2097-2100.

54 Cooley S, Trachtenberg E, Bergemann TL, Saeteurn K, Klein J, Le CT, et al: Donors with group B KIR haplotypes improve relapse-free survival after unrelated hematopoietic cell transplantation for acute myelogenous leukemia. Blood 2009;113:726-732.

55 Venstrom JM, Pittari G, Gooley TA, Chewning JH, Spellman S, Haagenson M, et al: HLA$\mathrm{C}$-dependent prevention of leukemia relapse by donor activating KIR2DS1. N Engl J Med 2012;367:805-816.
56 Miller JS, Soignier Y, Panoskaltsis-Mortari A, McNearney SA, Yun GH, Fautsch SK, et al: Successful adoptive transfer and in vivo expansion of human haploidentical NK cells in patients with cancer. Blood 2005;105:30513057.

57 Rubnitz JE, Inaba H, Ribeiro RC, Pounds S, Rooney B, Bell T, et al: NKAML: a pilot study to determine the safety and feasibility of haploidentical natural killer cell transplantation in childhood acute myeloid leukemia. J Clin Oncol 2010;28:955-959.

58 Leong J, Chase J, Romee R, Schneider S, Sullivan R, Fehniger T: Cytokine activation induces $\mathrm{CD} 25$ expression and a functional highaffinity IL-2 receptor on CD56 ${ }^{\mathrm{dim}}$ human NK cells. J Immunol 2014;190:183.10. http:// jimmunol.org/cgi/content/meeting _ abstract/190/1_MeetingAbstracts/183.10.

59 Kim S, Poursine-Laurent J, Truscott SM, Lybarger L, Song Y-J, Yang L, et al: Licensing of natural killer cells by host major histocompatibility complex class I molecules. Nature 2005; 436:709-713

60 Luetke-Eversloh M, Hammer Q, Durek P, Nordstrom K, Gasparoni G, Pink M, et al: Human cytomegalovirus drives epigenetic imprinting of the IFNG locus in NKG2Chi natural killer cells. PLoS Pathog 2014;10: e1004441.

61 Schlums H, Cichocki F, Tesi B, Theorell J, Beziat V, Holmes TD, et al: Cytomegalovirus infection drives adaptive epigenetic diversification of NK cells with altered signaling and effector function. Immunity 2015;42:443456.

62 Lee J, Zhang T, Hwang I, Kim A, Nitschke L, Kim M, et al: Epigenetic modification and antibody-dependent expansion of memorylike NK cells in human cytomegalovirus-infected individuals. Immunity 2015;42:431442 